\title{
Analytical chemistry for cultural heritage: a key discipline in conservation research
}

\author{
Rocco Mazzeo • Aldo Roda • Silvia Prati
}

Published online: 27 January 2011

(C) Springer-Verlag 2011

Conservation research can be defined as the work of scientists and others who pursue their investigations into the study and the conservation of cultural heritage. Conservation research as such is a form of applied research, which is focused on the conservation of material aspects of cultural heritage. It includes scientific studies where (art) historical, conservational and/or chemical, biological and physical methods are used. Therefore, conservation research is basically interdisciplinary in its approach.

Within this context, natural scientists apply both examinations of objects (works of art and science) using scientific methods as well as conservation science, which is a branch of natural science that is concerned with the physical/ material aspects of works of art and science, their deterioration and conservation. Scientific and technological disciplines, such as conservation science, rely on measurements of physical and chemical properties. Such measure-

Published in the special issue Analytical Chemistry for Cultural Heritage with Guest Editors Rocco Mazzeo, Silvia Prati and Aldo Roda.

R. Mazzeo $(\bowtie) \cdot$ S. Prati

Microchemistry and Microscopy Art Diagnostic Laboratory

(M2ADL), Alma Mater Studiorum-University of Bologna,

Via Guaccimanni 42,

48100 Ravenna, Italy

e-mail: rocco.mazzeo@unibo.it

S. Prati

e-mail: s.prati@unibo.it

\section{A. Roda}

Laboratory of Analytical and Bioanalytical Chemistry,

Department of Pharmaceutical Sciences,

Alma Mater Studiorum-University of Bologna,

Via Belmeloro 6,

40126 Bologna, Italy ments are central to, for example, analytical chemistry, that branch of chemistry concerned with determining the qualitative and quantitative identity of a substance.

Chemistry was first applied to the conservation field in the eighteenth century, gradually assuming a fundamental role, owing to the increased number of objects and collections exhibited in museums. Today, chemistry, and analytical chemistry in particular, plays a crucial role in the characterization of the nature of heritage materials, in the study of ancient production techniques, as well as supporting archaeometric studies (provenance, dating, attribution), detecting causes and mechanisms of degradation and developing and evaluating the performance of restoration materials and methods.

Ancient artworks, such as paintings, are complex heterogeneous systems often characterized by a multilayer structure. Therefore, the examination of artworks is often complicated by the difficulties in physically separating the different layers owing to their thickness $(1-200 \mu \mathrm{m})$. Moreover, a single layer is often composed of a mixture of various compounds both of organic and of inorganic nature. Such substances are not distributed homogeneously and numerous interactions between them, as well as degradation and ageing phenomena, may take place. To have a deepened understanding of these phenomena, advanced analytical techniques are therefore not only required at a molecular level to characterize the nature of the compounds but also to obtain chemical and physical information about ongoing changes. Another important issue to be taken into serious account is the need to reduce to a minimum the number and amount of samples collected from artworks. For this reason it is always advisable to follow a methodological approach where non-destructive and microdestructive techniques can be combined in an integrated way. 
In the last few years research efforts have been devoted to the development of non-destructive techniques [X-ray radiography and computer tomography, X-ray fluorescence (XRF), multispectral imaging system, portable Raman and Fourier transform infrared (FTIR) spectroscopy]. Even though they are extremely useful, because of their nondestructiveness and possibility to be used in situ, they cannot provide a detailed stratigraphic characterization of the areas analysed. In this regard microdestructive analytical techniques applied to sample cross-sections, which allow the identification and the contemporary spatial location of both the organic and the inorganic components, have been widely studied and developed. In particular, special attention has been paid to the development of stratigraphic analytical techniques capable of detecting organic substances. Indeed, their characterization is a more difficult task because of their higher chemical variability, low concentration and tendency to be degraded by internal and environmental factors. The 2008 special issue of Analytical and Bioanalytical Chemistry entitled "Characterization of Paintings" was actually devoted to this particular research issue.

During recent decades, the interest of the scientific community in conservation and restoration has increased owing to a growing understanding that the causes of deterioration, the characterization of the state of conservation and the development and testing of new conservationrestoration materials and methods are a priority for the correct safeguarding of cultural heritage. This trend is likely to continue as the scientific community involved in conservation is shifting its interest somewhat from the application of analytical techniques aimed at gaining profound knowledge of the constitution of heritage materials and of ancient production techniques, to problem-solving approaches that favour research studies aimed at providing feasible and lasting solutions to concrete conservation problems. The need for such a change comes mainly and directly from conservator-restorers who are confronted with real problems on a daily basis and thus ask for reliable solutions. It is the guest editors' opinion that this change should be further nurtured and this Special Issue is aimed in this direction by presenting advanced research in analytical chemistry for cultural heritage within a wider perspective.

This includes the development of new analytical protocols for the evaluation of the performance of innovative bioprotective treatments for ancient metals and the study of alteration processes which took place on modern materials, contemporary works of art, mortars and pigments. Of great interest are the papers dealing with the use of synchrotron radiation (SR)-based techniques (SR X-ray diffraction and SR-FTIR spectroscopy, X-ray absorption spectroscopy) as well as those related to the development of new biomolecular methods (ELISA, mitochondrial DNA, surface-enhanced Raman scattering nanotag-complexed antibodies) for protein recognition and localization, for the understanding of the biological origin of animal glues and for the development of a multiplexed chemiluminescent immunochemical imaging technique for the simultaneous localization of different proteins in paint cross-sections. Such techniques are able to provide information on the organic substances employed and, in perspective, may be suitable to support archeometric studies.

The development of new multianalytical approaches and protocols for the identification of heritage materials such as natural resins and colorants is also presented and discussed.

Traditional analytical techniques have been improved in terms of both new sample preparation procedures, which allowed a greater number of heritage materials to be examined, and interpretation of results thanks to the great support provided by multivariate analyses which allow the characterization and the chemical mapping and imaging of unknown substances to be better performed.

Another important research issue which is well presented in this Special Issue concerns the use of advanced nondestructive methods such as NMR and 2D imaging performed with a portable XRF instrument.

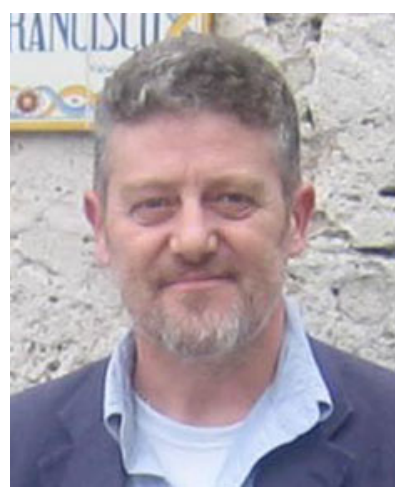

Rocco Mazzeo is Professor of Chemistry for Cultural Heritage at Bologna University, where he is actively involved in formal education in science for conservation and performs research in micro-FTIR mapping and imaging spectroscopic techniques applied to the study of painted works of art. 


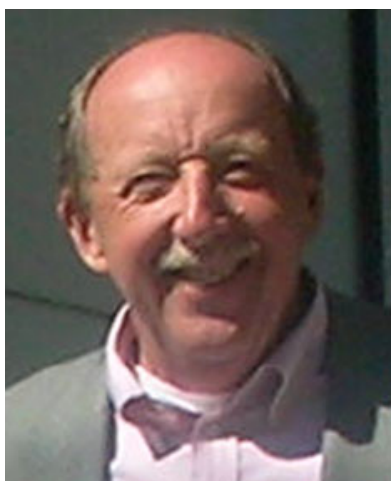

Aldo Roda is Professor of Analytical Chemistry at Bologna University. His main research interests include the development of ultrasensitive biochemiluminescence imaging techniques for the localization of target molecules (proteins, nucleic acids) in biological samples, such as cells and tissues.

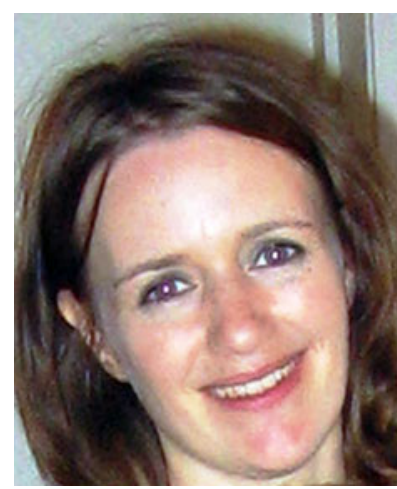

Silvia Prati is Researcher of Chemistry for Cultural Heritage at Bologna University, where she is actively involved in formal education in science for conservation and performs research in micro-FTIR mapping and imaging spectroscopic techniques applied to the study of painted works of art. 